\title{
Basics of continuous renal replacement therapy in pediatrics
}

\author{
Jacob C. John ${ }^{(\mathbb{1},}$, Sara Taha ${ }^{(1)}$, Timothy E. Bunchman ${ }^{(1)}$ \\ Department of Pediatric Nephrology, Children's Hospital of Richmond at the Virginia Commonwealth University, Richmond, VA, USA
}

\begin{abstract}
In the last three decades, significant advances have been made in the care of children requiring renal replacement therapy (RRT). The move from the use of only hemodialysis and peritoneal dialysis to continuous venovenous hemofiltration with or without dialysis (continuous renal replacement therapy, CRRT) has become a mainstay in many intensive care units. The move to CRRT is the result of greater clinical experience as well as advances in equipment, solutions, vascular access, and anticoagulation. CRRT is the mainstay of dialysis in pediatric intensive care unit (PICU) for critically ill children who often have hemodynamic compromise. The advantages of this modality include the ability to promote both solute and fluid clearance in a slow continuous manner. Though data exist suggesting that approximately $25 \%$ of children in any PICU may have some degree of renal insufficiency, the true need for RRT is approximately $4 \%$ of PICU admissions. This article will review the history as well as the progress being made in the provision of this care in children.
\end{abstract}

Keywords: Child, Kidney failure, Renal replacement therapy

\section{Introduction}

Since the initial work pioneered by Ronco et al [1] in the mid-1980s, continuous renal replacement therapy (CRRT) has transformed from an adapted therapy into the standard of care in pediatric critical care nephrology. This initial work was performed a single case report in an infant requiring continuous arteriovenous hemofiltra-

Received May 29, 2019; Revised July 23, 2019;

Accepted August 16, 2019

Edited by Gheun-Ho Kim, Hanyang University, Seoul, Republic of Korea Correspondence: Timothy E. Bunchman

Department of Pediatric Nephrology, Children's Hospital of Richmond at the Virginia Commonwealth University, 1000 E Broad St, PO Box 980498, Richmond VA 23298, USA. E-mail: Timothy.bunchman@vcuhealth.org

Copyright (c) 2019 by The Korean Society of Nephrology

(a) This is an open-access article distributed under the terms of the Creative Commons Attribution Non-Commercial License (http://creativecommons. org/licenses/by-nc-nd/4.0/), which permits unrestricted non-commercial use, distribution, and reproduction in any medium, provided the original work is properly cited. tion (CAVH). Notably, this method of renal replacement delivery was fraught with issues of inadequate blood flow rates (BFRs) due to its dependence on patients' cardiac output. With advances in vascular access and the novel capabilities of newer machines, $\mathrm{CAVH}$ has been substituted and replaced by continuous venovenous hemofiltration (CVVH). This method of RRT allows for clearance to occur independently of cardiac output; therefore, the indications for and populations able to use this modality have expanded [2].

During CRRT technology's infancy, most procedures were performed using adapted equipment. Then, as companies began investing in renal replacement technologies for pediatric patients in the mid-1990s, the use of CRRT in this population became more commonplace and more standardized equipment continued to become available. This article will offer a background on the terminology, equipment, basic theory, usage, and outcomes of CRRT.

The original equipment used worldwide was produced by Gambro (now under the banner of Baxter Interna- 
tional, Deerfield, IL, USA). Due to these initial efforts and as CRRT increasingly became the standard of care, many other companies worked to introduce CRRT systems. As a result, there are now many machines available throughout Asia, Europe, Australia, and North America.

Regardless of the machine used, however, considerations pertaining to equipment selection for CRRT include decisions on membranes, tubing, and vascular access. Matching the membrane surface area to the patient surface area is important to ensure adequate clearance. Membrane surface areas range from as small as $0.02 \mathrm{~m}^{2}$ to roughly $1.4 \mathrm{~m}^{2}$. This allows for adaptations to be made for the smallest of children to very large individuals. Considerations for membrane and tubing volumes should be made with regard to circulating blood volumes to avoid extraneous blood loss in these patients, as they are prone to anemia. The tubing and membranes of these systems are available as independent components (and tailored to an individual's needs) or as a combined cassette with a smallest extracorporeal circuit volume of $60 \mathrm{~mL}$ and a largest volume of nearly $250 \mathrm{~mL}$ [3].

Given the known problems with flow characteristics in smaller-caliber tubing and associated clotting issues, pediatric vascular access has long been a significant area of research. Many companies including Medcomp (Harleysville, PA, USA), Arrow Medical (Kington, UK), Covidien (Dublin, Ireland), and Cook Medical (Bloomington, IN, USA) now offer noncuffed access capabilities for acute dialysis modalities including CRRT. When choosing access, it is important to understand the maximum flow rates that different-sized catheters can achieve to ensure appropriate clearance. Vascular access sizing varies from 6 to 7 French-sized catheters used in smaller children to double- to triple-lumen 10 to 12 French-sized catheter in larger children. Catheter location is another factor affecting flow characteristics; Hackbarth et al [4] suggested that the optimal location for access in CRRT is the right internal jugular vein.

\section{Terms and theory}

Regardless of the machines used, one constant aspect is that they all abide by one or more of the principal tenants of mass transport, which include diffusive clearance (continuous venovenous hemodialysis; CVVHD), convective clearance $(\mathrm{CVVH})$, or a combination of convec- tion and diffusion (continuous venovenous hemodiafiltration).

Diffusion is the primary mechanism of transport with CVVHD. In this form of clearance, dialysate (a sterile physiologic solution) is administered on the opposing side of a semipermeable membrane to that of the patient's blood. Through random Brownian motion, solute equilibrates across the semipermeable membrane. Typically, dialysate is given counter-current to the blood flow, allowing for a sustained solute gradient for more efficient clearance. The same principle and mechanisms of using dialysate are seen in standard hemodialysis (HD), sustained lower-efficiently dialysis (SLED), and peritoneal dialysis (PD).

The use of CVVH is known as convective clearance. A physiologic, sterile solution is introduced in the vascular space either pre- or post-membrane in the CRRT circuit, and a pressure gradient is generated, promoting solvent flow through the membrane. The concept of convection is that, by mass transport, the solute is forced across the membrane by solvent drag rather than random Brownian motion associated with diffusion. With standard diffusion, as solutes approach the membrane pore size, the probability of random transport across the membrane becomes infinitesimally small. With solutes following a solvent across the membrane, the flow is more directed and middle molecules are more effectively cleared. The use of convection and diffusion together results in the concept of continuous venovenous hemodiafiltration. The choice of modality is dependent upon the clinical scenario presented and should be tailored to individual patients' treatment requirements.

Data by Maxvold et al [5] published in 2000 indicate that, for small-molecular-weight membranes, the convection and diffusion are identical for solute clearance. Experience in sepsis as well as cytokine responses identified that convection may be superior to diffusion in patients who are highly inflamed. The clearance of cytokines is nonspecific and circulating inhibitory cytokines are reduced, potentially minimizing the effects of proinflammatory cytokine removal. Furthermore, in areas of drug intoxications, convection is superior to diffusion because the molecule's sieving coefficient (SC) is greater in the convective mode. 


\section{Sieving coefficient}

The SC is a number assigned to indicate the rate at which the solute crosses the membrane. An example of this is that the SC of blood urea nitrogen (BUN) is 1 either in a convective or a diffusive mode. A sample of solute that is larger in size or that presents a greater affinity to protein binding will have superior clearance in a convective modality than in a dialysis modality. An example of this is vancomycin, which has an SC of 0.86 in CVVH but only 0.76 in CVVHD.

\section{Solutions}

Over the last 20 plus years, significant adaptations of solutions used for both convection and diffusion have been noted. Historically, these solutions were lactatebased, but data have since demonstrated that they deliver a lactate load to the patient, resulting in rising plasma lactate levels, which may falsely indicate worsening sepsis or poor perfusion states. Initial work done by Dialysis Solutions Inc. (Canada) produced a drug formally called Normocarb, which was the first bicarbonate-based solution used in North America [6]. This prototype solution has since become the standard of care throughout the world. Modern physiologic solutions include bicarbonate levels of 22 to $35 \mathrm{mmol} / \mathrm{dL}$ depending on the needs of the patient. Often, these include physiologically normal sodium levels, 0 to $5 \mathrm{mmol} / \mathrm{dL}$ of potassium, 0 to $3.5 \mathrm{mg} /$ $\mathrm{L}$ of calcium, and 0 to physiologically normal levels of phosphorus, and no urea (BUN). The use of calcium-free versus calcium-rich solutions is based on the concept of what type of anticoagulation is being used (citrate-based anticoagulation requiring calcium-free solutions).

The United States Food Drug Administration has identified convective solutions as a drug, while diffusive solutions are considered as a device. Therefore, in the United States, dialysate solutions are not allowed to be used for convection, yet convection solutions can be used for diffusion.

\section{Anticoagulation}

Anticoagulation options available are heparin, citrate, prostacyclin, or no anticoagulation. Many papers including work by Brophy et al [7], demonstrate that the no- anticoagulation protocol results in a very short life of the circuit. Historically, from the 1980s and 1990s, heparin was the standard of care, often used with a bolus of 20 unit/ $\mathrm{kg}$ followed by a continuous infusion at approximately the same dosage of $20 \mathrm{unit} / \mathrm{kg} /$ hour with a target bedside activated clotting time of roughly 200 seconds [8]. Brophy et al [7] pointed out that heparin-induced thrombocytopenia (HIT) occurred in approximately $3 \%$ of the population of a large retrospective study comparing heparin to citrate. Primary risk factors for HIT include previous exposure to heparin. Therefore, a patient's HIT risk needs to be monitored. Protocols for this can be seen on our website (www.pcrrt.com).

Initial investigations by Mehta et al [9] in adults and those in our children group demonstrate that citrate may be superior to heparin [10]. Citrate allows for localized anticoagulation of the circuit without systemic anticoagulation of the patient, which enables the achievement of a sustained circuit life without an increased risk of bleeding in the patient. Citrate is infused into the CRRT circuit after the blood leaves the patient but before the blood enters the CRRT filter. This results in chelating the calcium in the blood, therefore making the circuit hypocoagulable [6]. Calcium is then infused back into the patient via a central line independent of the circuit to reverse the anticoagulation and potential hypocalcemia that can occur with the administration of citrate. Citrate administration can cause metabolic alkalosis and calcium perturbations, which are readily manageable with attention to detail. More than 15 years ago, our group published the original and now widely used protocol for citrate anticoagulation in children [10].

Deep et al [11] performed research with prostacyclinbased anticoagulation in children with fulminant hepatic failure. Their institutional data suggest that prostacyclin is superior to other forms of anticoagulation with a circuit life of 1 to 2 days to avoid the risk of anticoagulation in the patient.

\section{CRRT prescriptions}

Historically a prescription for CRRT included a BFR of 3 to $5 \mathrm{~mL} / \mathrm{kg} / \mathrm{min}$, a dialysate or a replacement rate of 2,000 $\mathrm{mL} / 1.73 \mathrm{~m}^{2} /$ hour, and a net ultrafiltration rate of 0.5 to 2 $\mathrm{mL} / \mathrm{kg} / \mathrm{hour}[5,8]$. In reality, BFR is vascular access dependent. The greater the BFR is, the lesser the risk of clot- 
ting. The only contraindication of excessive BFR is if the child is at risk for rapid osmolar shift (dialysis disequilibrium) with associated high BUN, sodium, or glucose levels; in this setting, a slower BFR may be indicated [12]. The dialysate of replacement flow rates range from that noted above to Ronco's concept of $40 \mathrm{~mL} / \mathrm{kg} /$ hour [13]. These are starting points, and the amount of solution exposure (i.e., dialysate or replacement) can be increased or decreased based upon the solute clearance required.

\section{Blood priming in CRRT}

Currently, CRRT circuits range in extracorporeal volumes from $60 \mathrm{~mL}$ to $>250 \mathrm{~mL}$. Advances in smaller circuits (see later in the paper) may make standard-of-care CRRT circuits even smaller. The smaller the child is, the greater the need for blood priming. Historically, the idea of the $10 \%$ rule has been discussed. This suggests that, if the extracorporeal blood volume is $>10 \%$ of the intravascular blood volume of the child, then blood priming may be required [8]. However, work by Brophy et al [14] identified that if blood priming is performed, two innate risks may be present. If the CRRT circuit has an AN-69 membrane and if blood bank blood is quite acidotic $(\mathrm{pH}$ of 6.4), blood priming may interact with the AN-69 membrane, causing anaphylaxis at CRRT initiation. Additionally, blood bank blood is acidotic as well as hypokalemic and hyperkalemic, raising the risk of the blood causing a hyperkalemic arrest. The use of a bypass maneuver could mitigate the AN-69 risk but not the acidotic/hyperkalemic risk of the blood bank blood itself. Hackbarth et al [15] demonstrated the concept of "dialyzing the circuit" as a way to mitigate both of these risks. Essentially, what they presented involved blood priming but then, after dialyzing for a period of time, the $\mathrm{pH}$ would normalize and the risk of an AN-69 membrane reaction would lessen. The use of the AN-69 membrane is rare outside of the United States and has been substituted with a polysulphone membrane, which does not carry the same anaphylaxis risk.

\section{CRRT with extracorporeal membrane oxygenation (ECMO)}

CRRT is commonly used as a way to treat or prevent fluid overload as well as to normalize solute in children on ECMO. The incidence of acute kidney injury (AKI) is approximately $40 \%$ in children on ECMO, while the need for RRT may be close to $5 \%$.

Two options for CRRT added to ECMO are utilized. One involves placing a hemofilter within the ECMO circuit that acts as a slow continuous ultrafiltration device and will only affect fluid overload. Adding a replacement fluid or a dialysate fluid to that with the use of intravenous pumps can be done, yet this process is inaccurate. Further, an ultrafiltration controller incorporating an intravenous pump to "inhibit" the ultrafiltration rate can be considered, but this has been reported to present an inaccuracy rate of up to $30 \%$.

Alternatively, the addition of a CRRT machine added to the ECMO circuit will allow for a more accurate delivery of replacement and/or dialysate fluid and additionally offer more precise ultrafiltration control. When the CRRT machine is used with the ECMO device, the BFR of the CRRT machine should be independent of the ECMO device. Further, the "arterial access" of the CRRT machine needs to be set to tolerate "positive pressure" as opposed to the usual "negative pressure," as the ECMO access will allow for a very low-resistance circuit.

The location of where one hooks up the free-flowing or the circuit CRRT machine is important. If one does this in parallel with the oxygenator, then the risk of bypassing the blood away from the oxygenator to the CRRT exists. Classically, the CRRT system draws blood postoxygenator and returns it to the "bladder" of the ECMO circuit or the prepump area of the ECMO circuit to allow for minimal "stealing" of blood from the ECMO circuit to the CRRT system [16].

\section{Complications of CRRT}

As with all forms of extracorporeal RRT, there are risks associated with the use of these therapies. Hypothermia can occur, including a greater risk of such in children weighing $<25 \mathrm{~kg}$. This is due to the presence of a large extracorporeal circuit (relative to smaller children's intravascular volume) outside of the body at room temperature. One noted concern is that this effect could mask fevers and perhaps affect hemodynamics. Mechanisms to reduce thermal losses include providing overhead warmers for the child, warmers for the CRRT circuit, and inline blood warmers. However, none of these approaches 
is consistently effective, so individual styles of practice will often dictate the nature of this intervention. Until extracorporeal circuits are made smaller with subsequent reduced heat loss, this will remain an ongoing challenge to address.

Medication clearance can occur to the point of being detrimental to the treatment of underlying conditions. Factors that affect medication clearance include volumes of distribution, molecular weight, and protein binding. Vasopressor agents such as dopamine, dobutamine, epinephrine, and norepinephrine are cleared significantly. On the opposite side of the spectrum, medications like milirinone may be retained in the body, resulting in hypotension. In addition to the size of the medication, the location of the medication infusion in relation to the CRRT circuit's vascular access may affect clearance. Therefore, paying attention to the location of medication infusion, with regard to the proximity to CRRT access, may be important. Membrane reactions have become less common now due to a move away from the AN-69 membrane marketed by Baxter (historically Gambro) as the M-60 and M-100. These membranes reacted to acidotic plasma (e.g., blood bank blood with a pH of 6.4), causing a bradykinin release reaction and potential anaphylaxis [14]. The novel polysulphone membranes do not share these risks, and therefore, if blood priming is needed at the initiation of CRRT, the risk is only caused by the undesired properties of the blood bank blood itself ( $\mathrm{pH}$ of 6.4, ionized calcium of $0.02 \mathrm{mmol} / \mathrm{L}$, and a potential potassium load of $40 \mathrm{mmol} / 300 \mathrm{~mL}$ of blood bank blood).

The risk of thrombosis from the vascular access is more common in femorally placed vascular lines as well as in smaller children. The femoral vessels are smaller than the internal jugular vessels, with an associated significant risk for thrombosis. Additionally, the smaller the child is, the smaller the size of the vein. The optimal vascular access for RRT is "as big as one can place for optimal blood flow"; thus, this places the smaller child at great risk due to the disproportionality of the vascular access to the vein.

CRRT circuit clotting occurs due to a variety of issues. To begin with, one should ensure that the vascular access allows for low-pressure "pull and push" of the blood within the "arterial" and "venous" access is important. If there is resistance at this level, then the clotting risk will increase and the consideration of declotting the access needs to be undertaken. Second, BFR needs to be as high as the vascular access allows (unless a risk of dialysis disequilibrium exists), targeting an "arterial access pressure" of about 50 to $150 \mathrm{mmHg}$ and a "venous access pressure" of 50 to $150 \mathrm{mmHg}$. Third, excessive ultrafiltration will result in clotting. If one looks at the hemofilter in a convective mode (CVVH), one can see that the bloodentry end of the hemofilter has diluted blood and the exit end of the hemofilter has hemoconcentrated blood. If one looks at the same rate of delivery of prefilter replacement fluid versus postfilter replacement fluid, the prefilter replacement fluid will allow for less clotting due to the amount of dilution throughout, while the hemofilter is greater than when the fluid is given postfilter. Further, if clotting is an ongoing risk and if the goal is small molecular-weight solute clearance (e.g., urea), then using all of the fluid as dialysate will not have as much of an impact upon hemoconcentration at the distal end of the hemofilter as compared with convective clearance.

\section{Applications of CRRT}

Indications for CRRT include various forms of AKI with or without fluid overload and with or without electrolyte disturbance. Further indications would be ammoniaproducing inborn error of metabolism (IEM) or patients with intoxications [17-19].

The most common cause of AKI in an intensive care unit setting is sepsis. In this setting, convective clearance may have superiority over diffusion. Data to date have not suggested CRRT to be either superior or inferior to any other mode of RRT for AKI. The comparisons of PD vs. HD vs. SLED vs. CRRT have never been completed in a prospective manner. Therefore, the use of modality is based on the style of practice and one's own experiences.

With ammonia-producing IEM, one can use CVVH or CVHD as a modality, either in sequence after HD or instead of HD. Problems with ammonia-producing IEM arise from the unknown generation rate of ammonia; therefore, CRRT may not give adequate clearance, making HD superior. Our personal experience is that HD is superior to CRRT because it is the largest source of solute clearance over a set time frame and can be used as sequential therapy with CRRT to mitigate complications of elevated ammonia levels prior to the control of ammonia 
Table 1. Comparison of RRT modalities

\begin{tabular}{|c|c|c|c|c|}
\hline Modality & CRRT & SLED & HD (standard or high flux) & PD \\
\hline BFR & $\begin{array}{l}\text { 3-5 } \mathrm{mL} / \mathrm{kg} / \mathrm{min} \\
\text { access dependent }\end{array}$ & $\begin{array}{l}3-5 \mathrm{~mL} / \mathrm{kg} / \mathrm{min} \\
\text { access dependent }\end{array}$ & $\begin{array}{l}3-5 \mathrm{~mL} / \mathrm{kg} / \mathrm{min} \\
\text { access dependent }\end{array}$ & $10-20 \mathrm{~mL} / \mathrm{kg} /$ pass $^{\mathrm{a}}$ \\
\hline Dialysis flow rate (liter/hr) & $0-4$ & 6 & $30-50$ & $0.5-2$ \\
\hline Convective flow rate (liter/hr) & $0-4$ & 0 & 0 & 0 \\
\hline Systemic anticoagulation & Heparin or citrate & Heparin or citrate & Heparin or none & None \\
\hline Thermic control & Yes & Yes & Yes & Partial \\
\hline Ultrafiltration control & Yes & Yes & Yes & Partial \\
\hline Solutions & Industry made & On line production & On line production & Industry made \\
\hline Drug clearance & Continuous & Intermittent & Intermittent & Continuous \\
\hline Nutritional clearance & Continuous & Intermittent & Intermittent & Continuous \\
\hline Solute clearance & 2 & 3 & 1 & 4 \\
\hline UF with hemodynamic stability & 1 & 3 & 4 & 2 \\
\hline
\end{tabular}

BFR, blood flow rate; CRRT, continuous renal replacement therapy; HD, hemodialysis; PD, peritoneal dialysis; RRT, renal replacement therapy; SLED, sustained lowerefficiently dialysis; UF, ultrafiltration.

${ }^{\mathrm{a}}$ This is pass per volume per $\mathrm{kg}$ not blood flow but gives an idea of the PD prescription.

generation [18].

With cases of intoxication, HD is clearly superior to CRRT because of the large volume of dialysate moving across the membrane (resulting in greater sustained solute gradient), but in patients who are hemodynamically unstable, CRRT may be necessary. In such individuals, CVVH or CVHD can be used. In theory, convection is superior to dialysate due to a better clearance outcome based upon SCs of solute. Work by our group has demonstrated that the use of sequential therapy of HD followed by CRRT for both IEM as well as intoxication maximizes rapid clearance and minimizes rebound. This model is easily adaptable at bedside using the same vascular access for both CRRT as well as HD [20,21].

Recent advances are being made by Ronco with the use of CARPEDIUM and Couthard with the use of NIDUS, and work is currently ongoing in Japan on the subject of using smaller and smaller blood volumes for CRRT [3]. The CARPEDIUM has a $27 \mathrm{~mL}$ extracorporeal circuit, while the NIDUS has a $14 \mathrm{~mL}$ circuit. Work by colleagues in Japan is focusing on a prototype machine that functions with as low as 3 to $5 \mathrm{~mL}$ of extracorporeal blood. These smaller circuits will require a more dedicated access point as demonstrated recently [22].

\section{Comparison with other forms of RRT}

Table 1 compares CRRT to other forms of RRT including PD, SLED, and HD. Like many areas of clinical care, no single modality is always correct, but understanding the benefits and risks of each modality is important.

\section{Conclusion}

In summary, since the original work published by Ronco et al [1] more than 30 years ago, there has been an increase in the use of CRRT due to improved equipment, vascular access, anticoagulation protocols, solutions, and warming devices. CRRT has been adapted over the last 25 years into an accessible bedside therapy. However, it remains user-intense with high expenses from the nursing and resources points of view when compared with other forms of dialysis. To date, there are no prospective data in children showing the superiority or inferiority of CRRT in comparison with any other form of RRT. This area of research should be explored further in the future.

\section{Conflicts of interest}

All authors have no conflicts of interest to declare.

\section{Authors' contributions}

Timothy E. Bunchman did the orginial draft and approved the final draft. Jacob C. John expanded the work an added in siginficant depth to the paper. Sara Taha help with references and worked with Jacob C. John on the final version. 


\section{References}

[1] Ronco C, Brendolan A, Bragantini L, et al. Treatment of acute renal failure in newborns by continuous arteriovenous hemofiltration. Kidney Int 1986;29:908-915.

[2] Bunchman TE, Maxvold NJ, Kershaw DB, Sedman AB, Custer JR. Continuous venovenous hemodiafiltration in infants and children. Am J Kidney Dis 1995;25:17-21.

[3] Clark WR, Villa G, Neri M, Ronco C. Advances in machine technology. Contrib Nephrol 2018;194:80-89.

[4] Hackbarth R, Bunchman TE, Chua AN, et al. The effect of vascular access location and size on circuit survival in pediatric continuous renal replacement therapy: a report from the PPCRRT registry. Int J Artif Organs 2007;30:1116-1121.

[5] Maxvold NJ, Smoyer WE, Custer JR, Bunchman TE. Amino acid loss and nitrogen balance in critically ill children with acute renal failure: a prospective comparison between classic hemofiltration and hemofiltration with dialysis. Crit Care Med 2000;28:1161-1165.

[6] Bunchman TE, Maxvold NJ, Barnett J, Hutchings A, Benfield MR. Pediatric hemofiltration: normocarb dialysate solution with citrate anticoagulation. Pediatr Nephrol 2002; 17:150-154.

[7] Brophy PD, Somers MJ, Baum MA, et al. Multi-centre evaluation of anticoagulation in patients receiving continuous renal replacement therapy (CRRT). Nephrol Dial Transplant 2005;20:1416-1421.

[8] Bunchman TE, Donckerwolcke RA. Continuous arterialvenous diahemofiltration and continuous veno-venous diahemofiltration in infants and children. Pediatr Nephrol 1994;8:96-102.

[9] Mehta RL, McDonald BR, Aguilar MM, Ward DM. Regional citrate anticoagulation for continuous arteriovenous hemodialysis in critically ill patients. Kidney Int 1990;38:976981.

[10] Bunchman TE, Maxvold NJ, Brophy PD. Pediatric convective hemofiltration: normocarb replacement fluid and citrate anticoagulation. Am J Kidney Dis 2003;42:1248-1252.

[11] Deep A, Zoha M, Dutta Kukreja P. Prostacyclin as an anticoagulant for continuous renal replacement therapy in children. Blood Purif 2017;43:279-289.

[12] Bunchman TE, Hackbarth RM, Maxvold NJ, Winters JW, Barletta GM. Prevention of dialysis disequilibrium by use of CVVH. Int J Artif Organs 2007;30:441-444.

[13] Ronco C, Bellomo R, Homel P, et al. Effects of different doses in continuous veno-venous haemofiltration on outcomes of acute renal failure: a prospective randomised trial. Lancet 2000;356:26-30.

[14] Brophy PD, Mottes TA, Kudelka TL, et al. AN-69 membrane reactions are $\mathrm{pH}$-dependent and preventable. Am J Kidney Dis 2001;38:173-178.

[15] Hackbarth RM, Eding D, Gianoli Smith C, Koch A, Sanfilippo DJ, Bunchman TE. Zero balance ultrafiltration (Z-BUF) in blood-primed CRRT circuits achieves electrolyte and acid-base homeostasis prior to patient connection. Pediatr Nephrol 2005;20:1328-1333.

[16] Smoyer WE, Maxvold NJ, Remenapp R, Bunchman TE. Renal replacement therapy. In: Fuhrman BP, Zimmerman JJ, eds. Pediatric critical care. 2nd ed. St. Louis: Mosby, 1998

[17] Barletta GM, Bunchman TE. Acute renal failure in children and infants. Curr Opin Crit Care 2004;10:499-504.

[18] McBryde KD, Kershaw DB, Bunchman TE, et al. Renal replacement therapy in the treatment of confirmed or suspected inborn errors of metabolism. J Pediatr 2006;148:770778.

[19] Bunchman TE, Ferris ME. Management of toxic ingestions with the use of renal replacement therapy. Pediatr Nephrol 2011;26:535-541.

[20] Bunchman TE, Barletta GM, Winters JW, Gardner JJ, Crumb TL, McBryde KD. Phenylacetate and benzoate clearance in a hyperammonemic infant on sequential hemodialysis and hemofiltration. Pediatr Nephrol 2007;22:1062-1065.

[21] Meyer RJ, Flynn JT, Brophy PD, et al. Hemodialysis followed by continuous hemofiltration for treatment of lithium intoxication in children. Am J Kidney Dis 2001;37:1044-1047.

[22] Garzotto F, Zaccaria M, Vidal E, et al. Choice of catheter size for infants in continuous renal replacement therapy: bigger is not always better. Pediatr Crit Care Med 2019;20:e170e179. 